\title{
Rejoinder on: Recent progress on the combinatorial diameter of polytopes and simplicial complexes
}

\author{
Francisco Santos
}

Published online: 1 October 2013

(C) Sociedad de Estadística e Investigación Operativa 2013

\section{To foresee the future we must consult the past}

Hiriart-Urruty reminds us of something I completely agree; in order to advance forward we need to look at the past and understand what others did. In this respect, let me mention that (as is hinted in Sect. 4.2) my counterexamples to the (bounded) Hirsch conjecture (F. Santos 2012) use two ideas from the 45-year old paper by Klee and Walkup (1967). (Unless otherwise stated, all references in this rejoinder refer to the bibliography in the main paper). On the one hand, the Strong $d$-step Lemma (Theorem 4.8) is an extension of the original $d$-step lemma of Klee and Walkup (Lemma 4.7). On the other hand, the initial constructions of 5-spindles of length 6 were inspired by the understanding of the Klee-Walkup unbounded non-Hirsch polyhedron in terms of the Cayley trick (see Fig. 11 in Kim and Santos 2009). However, I have to confess I have more than once been guilty of the sin that Hiriart-Urruty describes as everybody writes, nobody reads. And certainly, I agree with Hiriart-Urruty and Poincare that there are no solved problems.

The other three commentators, De Loera, Eisenbrad, and Terlaky, devote most of their commentaries to some of the things that I announce in the Introduction that I do not cover in my paper. I have to thank them for the effort of putting in a few pages so much interesting information. In a sense, I am glad not to have covered these topics, since that made them have to cover themselves, which they have done very nicely. Let me just add a couple of comments myself.

This rejoinder refers to the comments available at doi:10.1007/s11750-013-0291-y; doi:10.1007/s11750-013-0292-x; doi:10.1007/s11750-013-0293-9; doi:10.1007/s11750-013-0294-8.

F. Santos $(\bowtie)$

Departamento de Matemáticas, Estadística y Computación, Universidad de Cantabria, E-39005 Santander, Spain

e-mail: francisco.santos@unican.es 


\section{Criss-cross methods}

First, I have to apologize for not having even listed criss-cross methods as one of the topics "not covered." Criss-cross methods (see Fukuda et al. 1997; Fukuda and Terlaky 1997, 2000; Terlaky 1985, 1997 in Terlaky's comment and reference Friedmann 2011 in De Loera's) use pivoting in much the same manner as the simplex method does except they are allowed to walk away from the feasible region. They are relevant to the topic of my paper in two ways:

- On the one hand, as Terlaky points out, criss-cross methods are as good candidates as simplex methods in the quest for strongly polynomial LP (or, following Terlaky, LO) algorithms. Short (meaning, polynomially bounded) pivot methods for crisscross algorithms would be strongly polynomial.

- On the other hand, criss-cross methods illustrate very vividly that proving polynomial diameter bounds (that is, proving the polynomial Hirsch conjecture) would only be a step toward having a polynomial simplex algorithm. In criss-cross methods, the existence of short pivot sequences has been proved (Fukuda et al. 1997; Fukuda and Terlaky 2000 in Terlaky's comment) but still no algorithmic way to find them without first solving the linear program is known. Another example where this distinction is clear are the Klee-Minty cubes (Klee and Minty 1969); combinatorially they are just cubes (so they satisfy the Hirsch bound on the diameter), but geometrically they are designed to trick the Dantzig's pivot rule into visiting all the vertices of the cube before arriving to the optimum.

\section{Continuous analogies}

Terlaky and De Loera also coincide in emphasizing the recent "continuous Hirsch conjecture" of Deza et al. (2008) and related work. I cannot but agree that the analogies between the results and open problems in the two worlds ("diameter and simplex algorithms"/"curvature of central path and interior point algorithms") is striking. As an example, the paths of exponential curvature constructed in Deza et al. (2009) are an analogue of the Klee-Minty cubes I mention in the previous paragraph. Terlaky also mentions analogies of the $d$-step Theorem and of the existence (proved by Klee and Holt) of Hirsch-sharp polytopes for most values of $n$ and $d$. See De Loera 2011, Sect. 2.2, and Kim and Santos 2010, Sect. 2.5 (besides the original papers) for more details.

What is not clear to me is whether all these analogies between the continuous and the discrete are "just a coincidence" or whether there are deep reasons for them.

\section{Other topics and questions}

De Loera brings into the foreground several other very interesting topics related to the geometry of linear optimization. Among them:

- the existence of "bad" lower bounds for the worst-case behavior of basically all the pivot rules that have been proposed so far and, in particular, the exciting recent additions to the list (see Friedmann 2011 and Friedmann et al. 2011); 
- the study of "particularly structured" polytopes of interest in combinatorial optimization. For example, for transportation polytopes we know linear diameter bounds (see Borgwardt 2009; Chubanov 2013; Goffin 1982 in De Loera's comment) but not quite the Hirsch bound. This topic is also addressed by Eisenbrand.

Terlaky mentions also the problem that goes from the combinatorics of an algorithm to the actual computations. Computations have to be performed with either exact arithmetic (potentially leading to an explosion in coefficients) or in finite precision (leading to numerical analysis issues).

Acknowledgements Work of F. Santos is supported in part by the Spanish Ministry of Science (MICINN) through grant MTM2011-22792 and by the MICINN-ESF EUROCORES programme EuroGIGA-ComPoSe IP04-Project EUI-EURC-2011-4306.

I want to thank the four authors of commentaries for their time and the good work they did. I also want to thank Miguel Ángel Goberna and the journal TOP for the invitation to write this paper. I would very much have liked to extend my thanks to Jesús Artalejo, but between the writing of my paper and that of this rejoinder I received the extremely sad news of his sudden passing away. I wish to send my condolences to his friends, family, and colleagues. 